\title{
Methodology of Exoskeleton with Actuation Methods and Discussion of Nerves Sensing for Exoskeleton
}

\author{
Ayush Goyal', Madan Mohan Dhir', Vijay Kumar Gill' and Mohit Verma ${ }^{1,2,{ }^{*}}$ \\ 'Department of Electronics \& Communication Engineering, Manav Rachna University, \\ Faridabad121001, India; ayushoct29@gmail.com, madan.dhir218@gmail.com, vijay@mru.edu.in \\ 2Accendere CL Educate, New Delhi 110044, India; mohit.verma@accendere.co.in
}

\begin{abstract}
Background/objectives: As the use of rehabilitation exoskeletons (techniques) has become an important means of treatment of stroke patients. This study has discussed different actuator methodologies and techniques that can be used in a movement of the exoskeleton and proposed design of exoskeleton with enhanced efficiency. Methods/statistical analysis: After reviewing several actuation techniques and analyzing various products available in the market, a less complex easy functioning design of exoskeleton is proposed with improved performance and lifetime, which can able to detect the signal directly from the nerve system with the help of EMG sensors and command the actuator after processing of the signal for the required movement. Findings: Mainly there are two major contributors sensing and actuation technique that is responsible for the efficient working of the exoskeleton. There are such types of sensors available which can fetch data or signal directly from the nerve system of the human body. Tremendous work is going on to make the actuators less complex, handy and easy working in order to improve the quality of the exoskeleton. Again some of the conventional exoskeleton based on string and motor system is not appropriately performing. The electric linear actuation system is a better solution in order to make the exoskeleton more qualitative nowadays as compared to others. Improvements/ applications: Exoskeleton is helpful for the person with a disability in terms of movement and reduced their dependency on others. These devices are used to make a disabled person walking, running, jumping and do any other movementrelated work without any help.
\end{abstract}

Keywords: Exoskeleton, Sensor, Actuator, Nerve Impulse

\section{Introduction}

This new era is dedicated to the rehabilitation systems. Exoskeletons are becoming the new age rehabilitation systems. First, let us see what does exoskeleton means? An exoskeleton is a mechanical or electro-mechanical system/machine which a person can wear (similar to an externally wearable skeletal system) \& can act as a motion assistive device. Basically, exoskeletons are the mechanical/electro-mechanical systems which support the human motion. Nowadays, tremendous work is going on to enhance human-machine interaction. Therefore, the most convenient way to make a good interaction between a human and a machine is when a machine is made in such a way that it can enact a human motion. In order to achieve this machine should be smooth enough to detect the input of human motion directly from the human body. This paper presents machining, which is able to establish a good connection between a human and a machine i.e. exoskeleton.

${ }^{*}$ Author for correspondence 


\subsection{History}

The idea of the exoskeleton was made real in around the 1890s when Yagn's Running Aid was patented. The presented prototype was simple leaf spring, operating in parallel to the legs. It was intended for running \& jumping applications. 1 Then, in 1960 General Electric Research, Cornell University \& with the help of USONR (United States Office of Naval Research) Developed an Exoskeleton Prototype called HARDIMAN (Human Augmentation Research And Development Investigation)..,$\underline{3}$ In the 1970s era, Miomir Vukobratovic and his associates from the Mihailo Pupin Institute developed an Exoskeleton, which used hydraulic actuation for its hip, knee, \& ankle movement.,$\underline{5}$ Recently there is a good interest developed in this field and different exoskeletons and prototypes are developed for Rehabilitation of patients with spinal cord or brain injuries. Every exoskeleton developed has its own unique mechanism.

The application of exoskeleton in rehabilitation has started to become commercialized, due to some of the recently developed exoskeletons some of them use the Gait detection method, \& some use the EMG nerve sensing method or any other method for detection for the intended motion and direction of motion intended.

\subsection{Gait Recognition Technique}

It is defined as a technique that considers the walking style of an individual for identification. It is one of the biometric identification techniques like face, fingerprint, IRIS retina. As similar to these techniques, walking style is also considered as a set of the parameter to identify a specific individual. $\underline{6}$ In this technique gait of an individual is analyzed like static body parameter where height and distance are taken into consideration. ${ }^{?}$ Another sub-method of this technique is by dividing the human body parts into several sub-sections and analyzing them separately..

\subsection{EMG Nerve Sensing Method}

The basic principle behind this method is to detect the changes in the potential of the nerve when a nerve impulse from the brain is passed to a muscle. Thus, at first, we need to know how the nerve impulse gets transmitted from our brain to hand through nerves.

\section{Process of Transmission of Nerve Impulse}

\subsection{Generation and Conduction of Nerve Impulse}

\subsubsection{Electrical Process of Transmission}

There are 2 channels known as voltage-gated channel and leaked channel. The purpose of these channels is to generate potential difference which help polarization and depolarization of the nerve. There are 2 parts of the nerve outer and inner in which $\mathrm{k}^{+}, \mathrm{Na}^{+}, \mathrm{Ca}^{2+}$ and $\mathrm{Cl}^{-}$ions are present. These ions are responsible for the transmission of the nerve impulse from one neuron to another. The purpose of the ion channel is to pass a selective type of ions.

\subsubsection{Resting Potential:}

When there is no transmission of impulse, then the nerve is said to be at the resting stage. This stage is the polarization stage. In polarization $\mathrm{Na}^{+}$ions are present outside of the neuron and $\mathrm{k}^{+}$ion is present inside the neuron, which developed a potential difference between the layers as $3 \mathrm{Na}^{+}$ions are present outside the layer whereas $2 \mathrm{k}^{+}$ions are present inside as shown in Figure 1.

\subsubsection{Action Potential}

At action potential stage, a transmission of impulse takes place resulting in depolarization of the nerve. In depolarization, $\mathrm{Na}^{+}$ions are in fluxed into the membrane and $\mathrm{K}^{+}$ions are refluxed from the membrane, as a result, the polarity is reversed. The outer membrane is negatively charged, and the inner membrane is positively charged.

After depolarization, repolarization occurs. In this stage, the $\mathrm{Na}$ channel gates are closed after 0.5

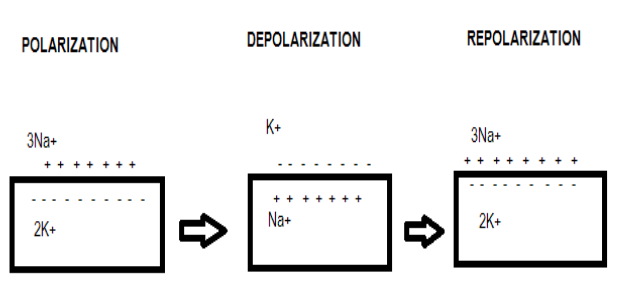

Figure 1. Representation of various stages. 
milliseconds. The $\mathrm{K}^{+}$is pumped in the membrane and $\mathrm{Na}^{+}$ions are pumped out of the membrane, as a result, the neuron comes back to the resting stage. This process of change from an excited state to the resting stage of the membrane is called repolarization, which exists for 1 to 5 milli seconds. When $\mathrm{K}^{+}$ions are induced in the membrane for a longer time period, it creates the inner potential more negative than usual; this is called hyperpolarization as shown in Figure 2.

\subsection{Propagation or Conduction of Nerve Impulse}

While the conduction of impulse, the resting potential does not propagate whereas the developed action potential propagates from one nerve to another. The process of conduction could be explained as shown in

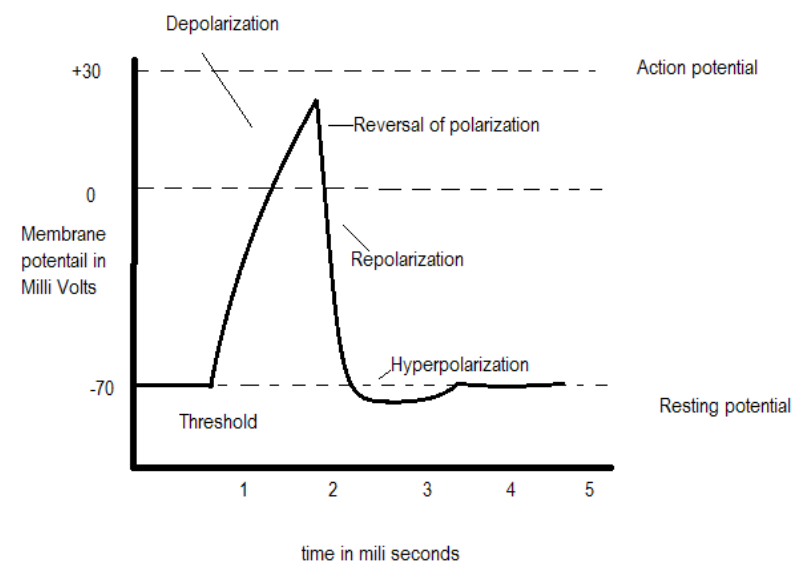

Figure 2. Representation of polarization, depolarization and depolarization state through potential.

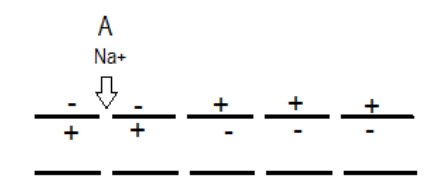

DEPOLARIZATION AT A

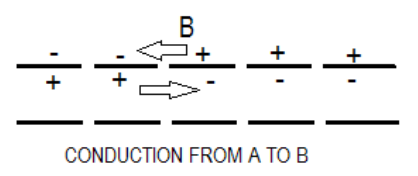

Figure 3. Schematic representation of depolarization and conduction state.
Figure 3. At point A depolarization takes place, the outer potential is negative as there consist $\mathrm{K}^{+}$ions whereas at point $\mathrm{B}$ there is resting potential developed as the outer membrane is positively charged due to the presence of the $\mathrm{Na}^{+}$ions and the inner membrane is negatively charged due to presence of less number of $\mathrm{K}^{+}$ion. The action potential develops in a site chain reaction in which the positive ions present at outside of the $B$ point travel from $B$ point to the negative site of A point due to the presence of potential difference where inside of the membrane, the conduction of charges takes place from the A point to $B$ point.

\subsection{Nerves Responsible for Hand Movement}

There are basically two components that constitute our nervous system named as Central Nervous System (CNS) and peripheral Nervous System (PNS). The CNS consists of the brain and spinal cord and the PNS consist of a bundle of nerves connecting the CNS to the other body part. Two-way communications take place between them in the coordination of the receptors which takes the stimulus from the environment and transfers it to the CNS through the PNS system. Now, this signal is processed inside the brain and action is generated. This generated action is then transferred to the PNS system which is connected to the muscles, in this way a command is performed and action is executed in coordination from the brain. $\underline{9}$

The nerve targeted in order to propose a prototype which is explained in this paper is given below:

Spinal accessory never is a type of cranial nerve which originates from the medulla and distributed at the shoulder region. It is a motor nerve that is responsible for the shoulder movement.

Brachial Plexus is a spinal nerve formed by the $5^{\text {th }} 6^{\text {th }} 7^{\text {th }}$ $8^{\text {th }}$ cervical spinal nerve and thoracic spinal nerve. It helps in arm movement.

Radial nerve is a part of brachial plexus. This nerve assists in the movement of the dorsal muscles.

\section{EMG Sensors}

The EMG sensors are used to take the input from the nerves of the arm muscles of a person. EMG sensors 
(electromyography sensors) allow the user to measure the electrical signal of the nerves and control this signal from an outside device. EMG sensors have Electrodes that are fitted on the surface of the arm of the individual. It will collect the data from the nerves. There will be three electrodes fitted on the arm, before attaching the electrodes, the dead cells on the skin are to be removed from it by applying some alcohol or its substitute, as they decrease the efficiency. The skin is then abraded lightly in order to increase the conductivity and these sensors sense accurate impulses from the nerves.

\section{Actuator Technology}

There are already many different types of actuators used for the same. Researchers worldwide are continuously working to design and develop specific actuators for exoskeletons in order to get accurate and efficient movement. Currently available actuators have some limitations, that they cannot able to provide the proper actuation without making the system more complex. Some of the actuators are discussed in this section.

\subsection{Electric Drives and Electric Linear Actuators}

When the electric drives term came, the first thought comes to mind is DC motors. DC motors are currently the most commonly used devices for achieving different mechanical actuation in the field of robotics. However, if the exoskeletons are specifically focused then the servomotors are the one who is most commonly used to develop a design. $\underline{10}-\underline{13}$ After motors, the electric linear actuators take away the attention. This helps to convert the rotational motion of a motor into the linear motion. These are very less bulky as compared to the hydraulic and pneumatics and need a very less maintenance cost. In this system, the motor provides the motion, which is then converted into linear motion by a lead screw running up and down. The motors and actuators are characterized by a number of properties and characteristics that are particularly important in the field of exoskeletons and robotics. Some of them include a good torque to weight ratio, high overload capacity, the ability to develop high torque when the motor shaft is stationary and in the speedy condition with the flexibility movement. In terms of the ease and flexibility of the installation electric actuators are much easier as compared to Hydraulics or Pneumatics. $\underline{14}$

\subsection{Pneumatic Actuators}

It consists of a piston that is encircled by a hollow cylinder. Pressure from an external compressor is responsible for the movement of the piston. As pressure is increased, it creates a force on the piston in a linear direction. The piston gets back to its initial position by a spring-back force. These are very cost-effective and have a high power to weight ratio. Also, these are simple to use, easy to maintain and have a long working life. $\underline{15}$ They generate a near to precise linear actuation but are less efficient due to the pressure losses and air's compressibility.

The compressor must run continuously whether there is a load or not. This makes them very bulky to use and a little bit inaccurate in nature.

\subsection{Hydraulic Actuators}

Hydraulic actuators work in a similar way as Pneumatic Actuators, but instead of pressurized air, an incompressible liquid is supplied to the piston, which makes it more strong then pneumatic actuators. These actuators are rugged and have high force strength. They produce a force 25 times greater as compared to the pneumatic actuators of the same size. They have a high horsepower to weight ration than pneumatic actuators. Electric and Hydraulic Actuators are competitive in terms of power to weight ratio, the capacity of the heavy load lifting etc.

\subsection{McKibben Artificial Muscles}

These types of artificial muscles are great when we talk about mimicking the actions of the muscles. These muscles require a medium to operate, either liquid or a gas medium. Their main advantage is that they have a high power to weight ratio along with the high power to volume ratio. Although the accuracy of these to control the muscles is limited due to the non-linearity of the compression of the medium. $\frac{16}{}$ Since they need a medium to operate and require enough supply of it, which then needs a compressor and an air handling unit, for gasoperated artificial muscles. This makes the system bulky, and the stability of the system is affected.

\section{Existing Designs}

There are many companies working to develop the exoskeleton, and some of these companies have even launched their products for sale. All these companies used different methods to sense the motion and produce 


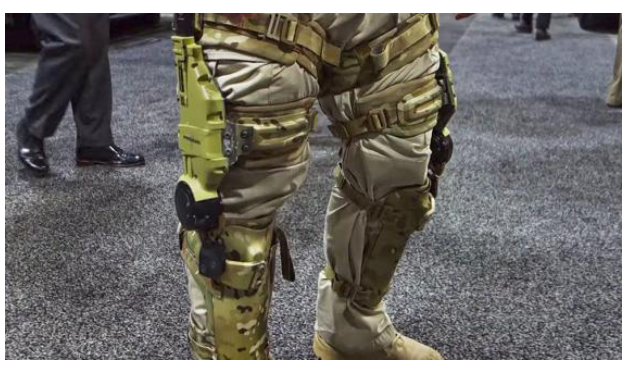

Figure 4. Human assistive system.

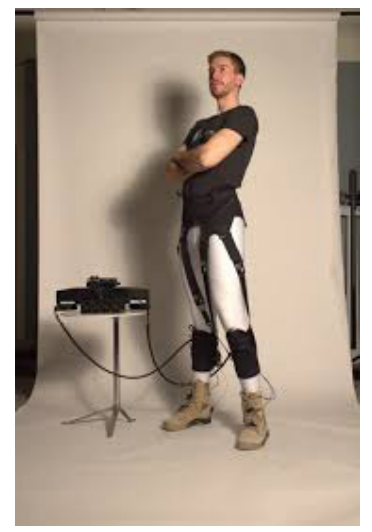

Figure 5. Soft Exo.

the actuation in order to make the exoskeleton. Some of them are described in Figures 4 and 5:

\subsection{Revision Military's Human Assistive System (HAS)}

It is a knee and hip exoskeleton. It is a partial exoskeleton with motorized knees only. The exoskeleton appears to be torque controlled only. As a wearer starts to move its knee in a direction, the processing unit of exoskeleton would slightly spin the motors and a signal will be associated which, depending on the sensitivity, could send a signal for the knee motor to spin. Motorized exoskeletons consume more of the power than linear magnetic actuators. They are also less reliable in terms of flexible movement. It is very inefficient in terms of flexibility, heavy weight lifting capabilities and smooth movements.

\subsection{Soft Exo (Harvard University)}

This is a wearable garment that provides assistance in movement by sending a motion to the wearer's joints. It partially assists the wearer's movements. But when it comes to the rigorous movements or uses, its performance is highly affected. Again, it is a cable-driven exoskeleton

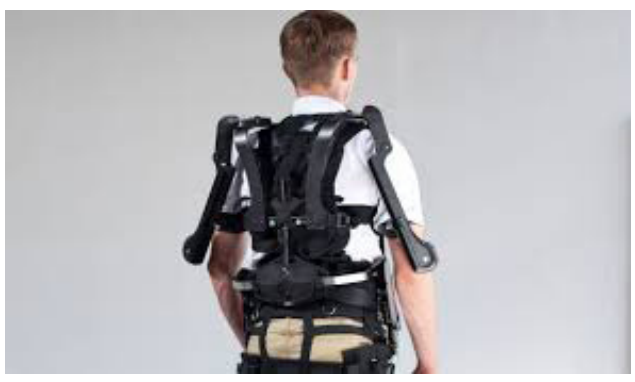

Figure 6. SuitX's modular agile exoskeleton.

which is not an effective method for actuation. Also, the reliability of this actuation method is not appropriate.

\subsection{SuitX's Modular Agile Exoskeleton (MAX)}

It is intended to assist the workers involved in intense labour work. It can support bending and lifting, it reduces the stress of the muscles of the wearers as shown in Figure 6.

\section{Proposed Design}

In this proposed design, an external assist system is planned to design which helps an individual to lift heavy loads. This design gives the Individual external support system to perform heavy load lifting tasks for a longer duration of time as well as with fewer efforts as shown in Figure 7.

\subsection{Component Details}

\subsubsection{Electric Linear Actuator}

It is of 4-inch stroke and $560 \mathrm{lb}$ thrust super duty linear actuator with 6-12 volt operating voltage. It weighs about $142.3 \mathrm{oz} / 4 \mathrm{~kg}$ and the current drain is approximately 3 amp. It has the ability to withstand a static load of 3050 lbs / $1383.5 \mathrm{~kg}$ with a dynamic thrust of $560 \mathrm{lbs} / 254 \mathrm{~kg}$. No-load speeds up to 2.63 inches per second and max load speed up to 1.85 inches per second. It has a metal gear train with a straight cut spur gearbox style with having a gear ratio of 5:1. Ingress protection of IP54 lead screw is ball screw type and it is made of steel.

\subsubsection{EMG Sensor}

It operates at a $\pm 9 \mathrm{~V}$ dual power supply. It requires a minimum voltage of 3.5 volts for working. This sensor 


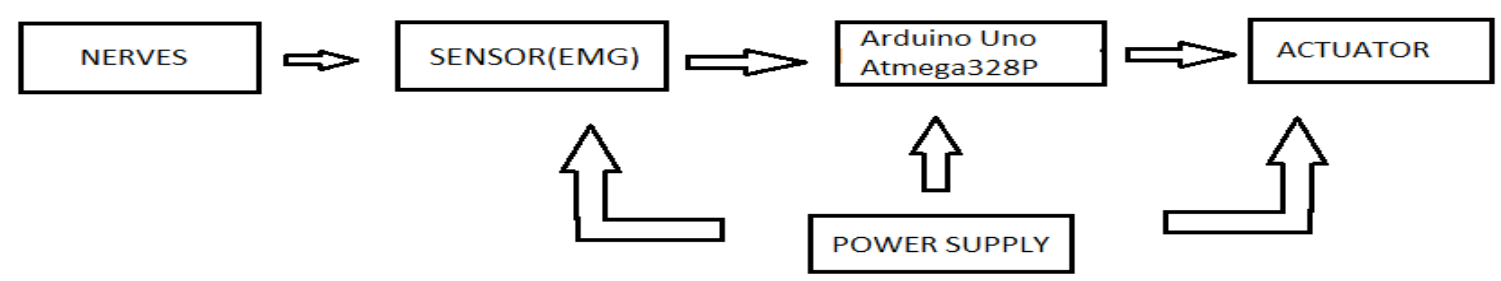

Figure 7. Block diagram of proposed design.

takes the input, performs filtering rectifying and amplification operation and generates the signal which is transferred in the Arduino. These sensors having small form factors with adjustable gain.

\subsubsection{Battery}

Lithium polymer 12 volt DC battery is used to power the actuator and 2 small lithium-ion batteries of 9 volts are connected in the series manner in order to power the EMG sensor and the Arduino as shown in the diagram.

\subsubsection{Arduino}

It is an Atmega328p 8 bit AVR family microcontroller which is operated at 5 volt and can withstand 7-12 volts of input (recommended). It contains 14 digital Input-output pin (6 are Pulse width modulation output) and 6 Analog input-output pins, the output current of these pins are 40 $\mathrm{mA}$ and $50 \mathrm{~mA}$ for 3.3 volt Pin. It contains Flash memory of $32 \mathrm{~KB}$ and SRAM $2 \mathrm{~KB}$, EEPROM $1 \mathrm{~KB}$ and operates at $16 \mathrm{Mhz}$ frequency.

\subsubsection{MC33886 Driver}

Supply Voltage $5 \mathrm{~V}-28 \mathrm{~V}$, operating frequency $10 \mathrm{kHz}$ continues current output $5 \mathrm{~A}$ an $\mathrm{H}$-Bridge Module Continuous DC Load Current up to 5.2 A.

The basic idea of the working procedure of the exoskeleton: In the proposed design the nerve impulse traveling in the nerve form EMG sensor is detected and processed. There are EMG electromyography sensors (with EMG electrodes) installed at some critical points on the arm to take the readings. These EMG electrodes are fitted on the specified nerve which carries the nerve impulse from the brain to the skeleton muscle. The initial reading for the action potential is about $+30 \mathrm{mV}$ whereas the resting potential is about $-70 \mathrm{mV}$. The reading is captured by the EMG sensors and feed to the module. This module performs rectification, amplification and filtering of the signal captured by the electrode and generates an analog signal which is transferred to the microcontroller that is Atmega328p which is calibrated in such a way that it detects the changes in potential and generates an output in accordance for the MC33886 motor driver. The motor driver takes the low current input from the microcontroller, amplifies the generated signal and feeds it to the actuator with the proper current and voltage required for the performing. Through this process, the proposed exoskeleton provides external assistance to the individual in order to lift heavy loads. This proposed design is not based on the motors or cable-driven, which is of less efficiency while performing. The used sensors are more accurate and rapid in sensing the movement/motion of the muscles. Again, this prototype uses a linear electric actuator instead of Pneumatic, or Hydraulic Systems, which make this system less complex and light in weight. The lifetime and the efficiency of the proposed exoskeleton are also improved as compared with the other available exoskeleton. Related font family throughout the article unless otherwise mentioned; the right margins should be justified wherever possible. You may also download the 'Indian Journals-article submission-model' for your convenience. Abbreviations should be expanded at least one time (initially) in the article as shown in Figure 8.

\section{Future Scope}

There are several works going on in order to improve the efficiency and performance of exoskeleton. Researchers 


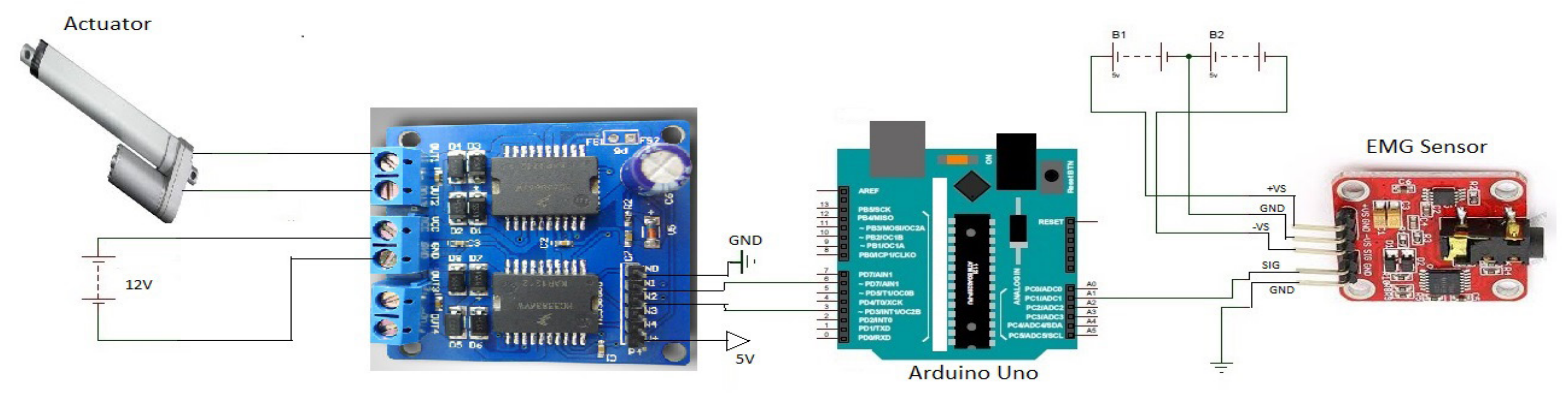

Figure 8. Circuit diagram representation of proposed design.

from worldwide are continuously working to enhance the various properties of an actuator such as the power to weight ratio, lower power consumption, making them compact etc. There is tremendous research going on to improve the efficiency and quality of the actuators to use in exoskeletons such as Mckibben Artificial Muscles,,$\underline{17}$ and Electromagnetic actuators. $\frac{18}{}$ As the actuator technologies become more efficient, better and compact, these actuators can be used in the future model of these exoskeletons in order to improve its performance and efficiency.

\section{Conclusion}

The Exoskeleton has proved themselves as a very helpful device for the person with disability in terms of movement. These devices make them self-organizing, controlled and increase their confidence. Again, these are helping to reduce their dependency on others. Nowadays, due to several accidents, these types of states can come for anybody and anywhere. In this paper, an exoskeleton is proposed with more accuracy and efficiency with lightweight and having more capability to lift loads. The designed exoskeleton is attached beside the arm and fetches the instructions from the nerve system itself, processed these signals and command for the movement as required. The proposed exoskeleton having a less complex structure and easy to use along with the lower maintenance factors.

\section{Acknowledgement}

Authors would like to express the gratitude to the Research Mentors of Accendere Knowledge Management Services Pvt. Ltd. for their comments on an earlier version of the manuscript. Although any errors are our own and should not tarnish the reputations of these esteemed persons.

\section{References}

1. Yagn N. Apparatus for facilitating walking, running, and jumping. U.S. Patents 420 179. 1890.

2. Ali H. Bionic exoskeleton: history, development and the future. In: International conference on advances in engineering \& technology: IOSR journal of mechanical and civil engineering; 2014. P. 58-62.

3. Fick BR, Makinson JB. Hardiman i prototype for machine augmentation of human strength and endurance. General Electric Co Schenectady NY specialty materials handling products operation; 1971.

4. Vukobratovic M, Hristic D, Stojiljkovic Z. Development of active anthropomorphic exoskeletons. Med Biol Eng. 1974;12(1):66-80.

5. Owen T. Scientific fundamentals of robotics 7-biped Locomotion by M. Vukobratovic B. Borovac, D. Surla and D. Stokic Springer-Verlag, Berlin1990, 349pages with index (DM 158.00; c.E 57.00). Robotica. 1990;8(4):325.

6. Witting J. Shape memory alloy actuators applied to biomimetic underwater robots. Neurotechnology for Biomimetic Robots; 2000.

7. Tomie M, Takiguchi A, Honda T, Yamasaki J. Turning performance of fish-type microrobot driven by external magnetic field. IEEE Trans Magn. 2005;41(10):4015-7.

8. Saotome H, Okubo T, Ikeda Y. A novel actuator with $\mathrm{Nd}-\mathrm{Fe}-\mathrm{B}$ magnets swimming in parallel to the magnetic field. IEEE Trans Magn. 2002;38(5):3009-11.

9. Yang Y, Yeo CK. Conceptual network model from sensory neurons to astrocytes of the human nervous system. IEEE Trans Biomed Eng. 2015;62(7):1843-52.

10. Garrec P, Friconneau JP, Measson Y, Perrot Y. ABLE, an innovative transparent exoskeleton for the upper-limb. 
In: 2008 IEEE/RSJ international conference on intelligent robots and systems; 2008. P. 1483-88.

11. Chen F, Yu Y, Ge Y, Sun J, Deng X. WPAL for enhancing human strength and endurance during walking. In: 2007 international conference on information acquisition; 2007. P. 487-91.

12. Pratt JE, Krupp BT, Morse CJ, Collins SH. The RoboKnee: an exoskeleton for enhancing strength and endurance during walking. In: IEEE international conference on robotics and automation. Proceedings. ICRA'04; 2004, vol. 3. P. 2430-35.

13. Aguirre-Ollinger G, Colgate JE, Peshkin MA, Goswami A. Design of an active one-degree-of-freedom lower-limb exoskeleton with inertia compensation. Int J Robot Res. 2011;30(4):486-99.

14. Roskam J, Rice M, Eysink H. A comparison of hydraulic, pneumatic, and electro-mechanical actuators for general aviation flight controls. SAE technical paper; 1979. P. 13.

15. Ali HI, Noor SB, Bashi SM, Marhaban MH. A review of pneumatic actuators (modeling and control). Aust J Basic Appl Sci. 2009;3(2):440-54.

16. Caldwell DG, Medrano-Cerda GA, Goodwin M. Control of pneumatic muscle actuators. IEEE Control Syst Mag. 1995;15(1):40-8.

17. Klute GK, Czerniecki JM, Hannaford B. McKibben artificial muscles: pneumatic actuators with biomechanical intelligence. In: 1999 IEEE/ASME international conference on advanced intelligent mechatronics; 1999. P. 221-6.

18. Zhu J, Lu H, Guo Y, Lin Z. Development of electromagnetic linear actuators for micro robots. In: 2008 international conference on electrical machines and systems; 2008. P. 3673-79. 